\title{
Outcomes of critically ill end-stage kidney disease patients who underwent major surgery
}

\author{
Peerawitch Petchmak ${ }^{1}$, Yuthapong Wongmahisorn ${ }^{1}$, Konlawij Trongtrakul ${ }^{\text {Corresp. } 2,3}$ \\ ${ }^{1}$ Department of Surgery, Faculty of Medicine Vajira Hospital, Navamindradhiraj University, Bangkok, Thailand \\ 2 Department of Medicine, Faculty of Medicine, Chiang Mai University, Chiang Mai, Thailand \\ 3 Department of Medicine, Faculty of Medicine Vajira Hospital, Navamindradhiraj University, Bangkok, Thailand \\ Corresponding Author: Konlawij Trongtrakul \\ Email address: konlawij@live.com
}

Purpose. End-stage kidney disease (ESKD) is a major worldwide health problem. Patients with ESKD are thought to have a significant risk for development of complications following an operation. However, the study of ESKD and its outcomes following major operations remains rare, particularly in critical illness. Therefore, this study aimed to demonstrate how the outcomes of ESKD patients were affected when they underwent a major operation and were admitted to the intensive care unit (ICU), compared with non-ESKD patients.

Methods. A retrospective matched case cohort study was conducted in 122 critically ill surgical patients who underwent a major operation and were admitted to the ICU, during 2013 and 2016. Sixty-one ESKD patients who required long-term dialysis were enrolled and compared with 61 matched non-ESKD patients. The matching criteria were the same interval of age ( \pm 5 years), gender, and type of operation. The ICU mortality was compared to the primary outcome of the study.

Results. Patients' baseline characteristics between ESKD and non-ESKD were similar to a priori matching criteria and other demographics, except for pre-existing diabetes mellitus and hypertension, which were found significantly more in ESKD ( $p=0.03$ and 0.04 , respectively). For operations, ESKD showed a higher grade of the American Society of Anesthesiologist (ASA) physical status $(p<0.001)$, but there were no differences for emergency surgery $(p=0.71)$ and duration of operation $(p=0.34)$. At ICU admission, the severity of illness measured by Sequential Organ Failure Assessment (SOFA) score was greater in ESKD ( $8.9 \pm 2.6$ vs $5.6 \pm 2.5 ; p<0.001)$. However, after eliminating renal domain, SOFA non-renal score was equivalent ( $5.7 \pm 2.2$ vs $5.2 \pm 2.3, p=0.16)$. The ICU mortality was significantly higher in surgical critically-ill patients with ESKD than non-ESKD ( $23 \%$ vs $5 \%, P=0.007$ ), along with hospital mortality rates ( $34 \%$ vs $10 \%, p=0.002)$. The multivariable logistic regression analyses adjusted for age and SOFA non-renal score demonstrated that ESKD had a significant association with ICU and hospital mortality 
$(\operatorname{adjOR}=5.59 ; 95 \% \mathrm{Cl}, 1.49-20.88, p=0.01$ and $\operatorname{adjOR}=4.55 ; 95 \% \mathrm{Cl}, 1.67-12.44, p=0.003$, respectively).

Conclusion. Patients who underwent a major operation and needed intensive care admission with pre-existing ESKD requiring long-term dialysis were associated with greater mortality than patients without ESKD. More careful assessment before, during, and after major surgical procedures should be performed in this group of patients to improve postoperative outcomes. 


\section{Outcomes of Critically III End-Stage Kidney Disease}

\section{Patients Who Underwent Major Surgery}

3 Peerawithc Petchmak ${ }^{1}$, Youthapong Wongmahisorn ${ }^{1}$, Konlawij Trongtrakul ${ }^{2,3}$

$4{ }^{1}$ Department of Surgery, Faculty of Medicine Vajira Hospital, Navamindradhiraj University,

5 Bangkok, Thailand

$6{ }^{2}$ Department of Medicine, Faculty of Medicine Vajira Hospital, Navamindradhiraj University,

7 Bangkok, Thailand

$8{ }^{3}$ Department of Medicine, Faculty of Medicine, Chiang Mai University, Chiang Mai, Thailand

10 Corresponding Author: Konlawij Trongtrakul2,3

11 Email address: konlawij@live.com 


\section{Abstract}

14 Purpose. End-stage kidney disease (ESKD) is a major worldwide health problem. Patients with 15 ESKD are thought to have a significant risk for development of complications following an 16 operation. However, the study of ESKD and its outcomes following major operations remains 17 rare, particularly in critical illness. Therefore, this study aims to demonstrate how the outcomes of ESKD patients are affected when they underwent a major operation and are admitted to the 19 intensive care unit (ICU), compare with non-ESKD patients.

20 Methods. A retrospective matched case cohort study was conducted in 122 critically ill surgical 21 patients who underwent a major operation and were admitted to the ICU, during 2013 and 2016.

22 Sixty-one ESKD patients who required long-term dialysis were enrolled and compared with 61 23 matched non-ESKD patients. The matching criteria were the same interval of age ( \pm 5 years), 24 gender, and type of operation. The ICU mortality was compared to the primary outcome of the 25 study. 
26 Results. Patients' baseline characteristics between ESKD and non-ESKD were similar to a priori

27 matching criteria and other demographics, except for pre-existing diabetes mellitus and

28 hypertension, which were found significantly more in ESKD ( $p=0.03$ and 0.04 , respectively). For 29 operations, ESKD showed a higher grade of the American Society of Anesthesiologist (ASA)

30 physical status $(\mathrm{p}<0.001)$, but there were no differences for emergency surgery $(\mathrm{p}=0.71)$ and

31 duration of operation ( $\mathrm{p}=0.34)$. At ICU admission, the severity of illness measured by Sequential

32 Organ Failure Assessment (SOFA) score was greater in $\operatorname{ESKD}(8.9 \pm 2.6$ vs $5.6 \pm 2.5, \mathrm{p}<0.001)$.

33 However, after eliminating renal domain, SOFA non-renal score was equivalent (5.7 \pm 2.2 vs 5.2

$34 \pm 2.3, \mathrm{p}=0.16)$. The ICU mortality was significantly higher in surgical critically-ill patients with

35 ESKD than non-ESKD (23\% vs 5\%, P=0.007), along with hospital mortality rates (34\% vs $10 \%$,

$36 \mathrm{p}=0.002$ ). The multivariable logistic regression analyses adjusted for age and SOFA non-renal

37 score demonstrated that ESKD had a significant association with ICU and hospital mortality

$38(\operatorname{adjOR}=5.59 ; 95 \% \mathrm{CI}, 1.49-20.88, \mathrm{p}=0.01$ and $\operatorname{adjOR}=4.55 ; 95 \% \mathrm{CI}, 1.67-12.44, \mathrm{p}=0.003$,

39 respectively).

40 Conclusion. Patients who underwent a major operation and needed intensive care admission

41 with pre-existing ESKD requiring long-term dialysis were associated with greater mortality than

42 patients without ESKD. More careful assessment before, during, and after major surgical

43 procedures should be performed in this group of patients to improve post-operative outcomes. 


\section{Introduction}

46 Chronic kidney disease (CKD) is a major worldwide public health problem (Couser et al.

47 2011; Hill et al. 2016; Ong-Ajyooth et al. 2009). Patients suffering from CKD may experience

48 uremia, anemia, cardiovascular diseases, and decreased quality of life (Couser et al. 2011; Jha et

49 al. 2013). They can also progress to long-term dialysis, kidney transplantation, or even death, when

50 they proceed to end stage kidney disease (ESKD).

51 The development of ESKD has been increasingly reported over the past decades (Collins

52 et al. 2014; Thammatacharee \& Suphanchaimat 2020). In the United States, data suggests that the

53 incidences of ESKD are projected to increase up to $11-18 \%$ by the year 2030 , compared to those

54 of 2015 (McCullough et al. 2019). This feature could be explained by the current situation of a

55 growing aging population (Goulding et al. 2003). Moreover, the predispositions of patients toward

56 ESKD have been increasing; including obesity (Hales et al. 2018), hypertension (Forouzanfar et

57 al. 2017), and diabetes mellitus (Geiss et al. 2014). Patients with ESKD are more likely to have

58 complications with cardiovascular diseases, gastrointestinal bleeding, malnutrition, or

59 immunosuppression (Bagshaw \& Uchino 2009; Chan \& Ostermann 2013; Sood et al. 2011; Uchino

60 et al. 2003). These complications mostly require hospitalization and create economic burdens

61 (Praditpornsilpa et al. 2011). An increase of incidences (1\% to 1996 to 3\% in 2010) of dialysis-

62 requiring acute kidney injury (AKI) in critically-ill surgical patients is found (Wald et al. 2015)

63 and is also likely to be found in critically-ill patients with pre-existing ESKD. Previous studies

64 have reported the incidence of intensive care unit (ICU) admissions of ESKD at around 1-11\%

65 (Apel et al. 2013; Bagshaw \& Uchino 2009; Hutchison et al. 2007; Jha et al. 2013; Strijack et al.

66 2009). In addition, this population has a greater chance of admission to the ICU (25 times) and is 
67 associated with a higher mortality rate than the general population (Hutchison et al. 2007; Jha et 68 al. 2013; Strijack et al. 2009).

69 Critically-ill patients with ESKD consume more ICU resources than those who were not 70 ESKD (Arulkumaran et al. 2013; Fidalgo \& Bagshaw 2014), particularly, the challenges of the 71 maintenance of regular dialysis, the proper management of fluids, and the regulation of metabolism 72 and electrolytes. Moreover, the EKSD patients have several negative impacts including longer 73 length of ICU stay and longer length of hospitalization (Fidalgo \& Bagshaw 2014) and may be 74 confronted with several long-term effects following intensive care discharge, for instance, 75 cardiovascular complications, malnutrition, and deconditioning (Fidalgo \& Bagshaw 2014). This trend of increasing ESKD patients and resulting complications highlights the need 77 for improved resource utilization systems within a limited resource environment, such as the 78 surgical ICU. Numerous barriers have been found to preclude proper ICU bed allocation (van 79 Sluisveld et al. 2017), particularly regarding emergency surgery (Hasan et al. 2020). Inevitably, 80 ESKD patients will in some cases acquire diseases requiring surgical treatment and be at risk of 81 becoming critically-ill. Surgical ICU is a specialized unit serving patients who undergo general 82 surgery related to acute care and life-threatening conditions. Care of critically-ill surgical patient with ESKD needs to be further understood in relation to their physiology being disrupted by 84 surgical intervention. For example, is there a greater risk of morbidity and mortality among critically-ill ESKD patients who have undergone major operations, even though current evidence about the surgical outcomes is lacking. A new study is needed to address these concerns. interventions may induce stressors to ESKD patients differently from medical problems, data related to this study have mostly been drawn is from mixed medical and surgical intensive care 
90 units (ICU), and includes limited patient profiles regarding type of operation and perioperative

91 information. The purpose of the present study, therefore, is to explain how the outcomes of

92 critically-ill ESKD patients who undergo major operations are affected when compared with

93 surgical critically-ill non-ESKD patients. It is hypothesized that critically-ill ESKD patients who

94 undergo major operations will be independently associated with a greater risk of mortality than

95 surgical critically-ill non-ESKD patients.

96

\section{Materials \& Methods}

98

99

100

101

102

103

104

105

106

107

108

109

110

111

112

The study was approved by the Institutional Review Board of the Faculty of Medicine Vajira Hospital, Navamindradhiraj University, Bangkok, Thailand (approval no. 088/57). Waiving of informed consent was allowed due to the minimal risk of the study and its retrospective nature.

We conducted a retrospective matched cohort study in critically-ill surgical patients with and without ESKD by searching for cases who had undergone a major operation and had been admitted to our 3 surgical ICUs (a total of 17 beds) during the period of January, 2013 through December, 2016.

Inclusion criteria are adult critically-ill surgical patients (age equal to 18 years and above) with and without ESKD who had undergone a major operation and had been admitted to the surgical ICU within the first 48 hours post-operatively. Patients transferred to the ICU due to serious medical conditions not related to any operation, or critically-ill surgical patients who underwent specific procedures related to $\mathrm{CKD} / \mathrm{ESKD}$, such as arteriovenous fistula, any dialysis access, or kidney transplantation, were excluded from the study.

To reduce selection bias, critically-ill surgical patients without a history of ESKD were matched to critically-ill ESKD patients a priori with the same three baseline characteristics. The 
113 matching characteristics included age interval ( \pm 5 years), gender, and type of operation, their

114 significance in determining higher surgical risk. Previous data from critically-ill patients

115 undergoing major operations show that age (older than 65), is independently associated with

116 mortality (Elia et al. 2013). Gender, one of the general baseline demographics, has been found as

117 a covariate of high mortality for females (Romo et al. 2004). In addition, greater severity of illness

118 at surgical ICU admission, as measured by the SOFA score is associated with higher mortality

119 rates (Pornwaragron et al. 2019).

120 Definitions

121 Critically-ill ESKD patients were defined as those requiring chronic dialysis of at least 6

122 weeks before ICU admission by either hemodialysis (HD) or peritoneal dialysis (PD) (Apel et al.

123 2013; Strijack et al. 2009). Some critically-ill surgical patients without ESKD might have pre-

124 existing renal dysfunction, so kidney disease staging was defined according to KDIGO-2012

125 criteria (Kidney Disease: Improving Global Outcomes (KDIGO) CKD Work Group. 2013). Major

126 operation was defined according to all procedures that required either general or regional

127 anesthesia. Major operations were done based on surgeon decision and standard practice

128 guidelines in the study center.

\section{Data collection}

130 Baseline characteristics including patient age, gender, body weight, height, body mass

131 index (BMI), and pre-existing comorbidities were extracted from electronic medical records. Pre-

132 existing comorbidities including the presence of diabetes mellitus, hypertension, dyslipidemia, 133 cardiovascular disease, cerebrovascular accident, and chronic kidney disease were recorded. The

134 histories of ESKD patients were reviewed for their mode of long-term dialysis, which included 135 HD and PD. 

collected, together with the patients' American Society of Anesthesiologists (ASA) physical status. In addition, we collected the operative data regarding whether surgery was emergency or elective, type of operation, and duration of operation.

The primary outcome of the study was ICU mortality. Hospital mortality, ICU co-

morbidities, and complications during the ICU stay, ICU length of stay, and hospital length of stay were reported as secondary outcomes.

\section{Sample size}

The sample size to enhance statistical power in our study was calculated according to the

157 patients with ESKD were randomly selected based on available retrospective data. In the non-

158 ESKD group, patients were selected according to the above-mentioned matching characteristics. 


\section{Statistical analysis}

160 Continuous variables were summarized as mean $(\mu)$ and standard deviation (SD) for

161

162 163

164

165

166

167

168

169

170

171

172

173

174

175

176

177

178

179

180

181

normally distributed data, or median and interquartile range 1 and 3 (IQR1-3) for non-normal distributed data. Categorical descriptive variables were summarized as frequency (n) and percentages $(\%)$.

Statistical analysis plan for comparing mean and medians were between the two groups using Student's t-test or Mann-Whitney U test, when appropriate. Categorical variables were analyzed with Fisher Exact test. We performed multivariable logistic regression analysis calculating Odds ratios (OR) and its 95\% confidence interval $(95 \% \mathrm{CI})$ for identifying how ESKD affected the primary outcome and adjusted by covariates. The overparameterization of the multivariable regression model is a concern, due to a limited sample size in this study. Generally, it is accepted that events per variable should be greater than 10 when generating a multivariable regression model (Deng et al. 2017; Steyerberg et al. 2011). If the number of deaths is 30 out of a total of 122 cases $(25 \%)$, no more than 3 variables are allowed to be included in the model. Moreover, the most commonly used criteria for the variable selection in the multivariable regression model is p-value of less than $0.10-0.20$ in the univariable analysis (Deng et al. 2017; Harris et al. 2015; Trongtrakul et al. 2019). However, a priori variable selection is accepted (Walter \& Tiemeier 2009). This study used significant variables that are associated with a greater mortality risk in surgical ICU, including age (Elia et al. 2013) and severity of illness measured by SOFA non-renal score (Pornwaragron et al. 2019) as adjusted variables to understand the association between ESKD and mortality. The STATA statistical software version 13.0 (StataCorp LP, College Station, TX, USA) was used for statistical analyses and p-value of less than 0.05 was considered statistically significant.

Peer) reviewing PDF | (2020:04:47724:2:0:CHECK 15 Mar 2021) 


\section{Results}

\section{Baseline characteristics of the study}

A total of 122 surgical critically-ill patients were included in the study, 61 critically-ill with

ESKD and 61 critically-ill without ESKD (Figure 1). Fifty-five ESKD patients were commenced on long-term HD, while another 6 patients were commenced on PD. All three matching characteristics were comparable between ESKD and non-ESKD. They were similar in age types of operation (all p>0.05) (Table 1).

cerebrovascular diseases, were similar between groups, except diabetes mellitus and hypertension, which were more prevalent in ESKD than non-ESKD (67\% vs $46 \%$, $p=0.03$ and $89 \%$ vs $72 \%$, $\mathrm{p}=0.04$, respectively). Moreover, at least $7 / 61(12 \%)$ of non-ESKD patients had known baseline renal dysfunction categorized as CKD stage 2-4. ESKD had no significant effect on the technique selected for anesthetizing $(p=0.49)$ or on the status of emergency surgery $(\mathrm{p}=0.71)$. The majority of operations were vascular related surgery (excluding arterio-venous graft or catheterization for long term dialysis), followed by cardiothoracic and gastrointestinal tract surgery. Between the two groups, we did not find any differences in the types of operation (all p>0.05) and duration of operation (4 hours [IQR 2,6 hours] vs 3.5 hours [IQR 2,5 hours], $\mathrm{p}=0.34$ ). 
205 calculation of serum creatinine from the renal domain of SOFA score, both groups had a non206 significant difference in organ dysfunction (SOFA non-renal score $=5.7 \pm 2.2$ vs $5.2 \pm 2.3$, $207 \mathrm{p}=0.16)$.

208 Primary and secondary outcomes

209 ICU mortality rates were significantly greater in ESKD than non-ESKD (23\% vs 5\%, 210 respectively; $\mathrm{p}=0.007)$. ESKD also affected hospital mortality $(34 \% \mathrm{vs} 10 \%, \mathrm{p}=0.002)$ as shown 211 in Table 2 and Figure 2. When comparing the modalities for long-term dialysis in ESKD, there 212 was no difference in risk for ICU mortality between HD and PD (14/55 [25\%] cases vs 0/6 [0\%], 213 respectively, $\mathrm{p}=0.19)$.

214 The median time of ICU length of stay was significantly longer in ESKD than non-ESKD 215 (3 days [IQR 2,12 days] vs 2 days [IQR 1,6 days], respectively, $\mathrm{p}=0.007$ ). Moreover, longer 216 hospital length of stay was also found (29 days [IQR 16,51 days] vs 20 days [IQR 14,34 days], 217 respectively; $p=0.03)$.

Table 2 also illustrates our patients' comorbidities during admission to the ICU. The 219 presence of sepsis and septic shock was the most common, followed by cardiovascular diseases, $22039 \%$ (47/122 cases), and 28\% (34/122 cases), respectively. Despite many complications or co221 morbidities during the ICU stay, there were no differences between ESKD and non-ESKD (all $222 \mathrm{p} \geq 0.05)$.

223

224 ESKD and risk of mortality

225

Table 3 shows the association between ESKD and its effect on outcomes. A greater risk of 226 ICU mortality was found $(\mathrm{OR}=5.76 ; 95 \% \mathrm{CI}, 1.56-21.24, \mathrm{p}=0.009)$. In addition, hospital mortality 227 dominated in ESKD groups $(\mathrm{OR}=4.81 ; 95 \% \mathrm{CI}, 1.78-13.01, \mathrm{p}=0.002)$. After adjusting for age and 
228 SOFA non-renal score, both ORs remained statistically significant (OR=5.59; 95\%CI, 1.49-20.88, $229 \mathrm{p}=0.01$ and $\mathrm{OR}=4.55 ; 95 \% \mathrm{CI}, 1.67-12.44, \mathrm{p}=0.003$, respectively).

231 Discussion

\section{Summary of the study}

The main finding of our study demonstrated that critically-ill surgical patients suffering 234 long-term dialysis from ESKD who experienced a major operation had around a 5-time greater 235 risk of ICU mortality and hospital mortality than critically-ill surgical patients without ESKD. Moreover, ESKD also had an impact on longer duration of ICU stay and hospital stay than non237 ESKD.

238

239

240

241

242

243

244

245

246

247 248 249 250

\section{Comparing to the previous study}

To the best of our knowledge, data regarding the effect of ESKD on critically-ill surgical patients' outcomes have scarcely been reported. An extensive search revealed the largest cohort of surgical intensive care patients from the study by Apel M, et al (Apel et al. 2013). The study specifically investigated surgical intensive care patients who underwent operation (same features as ours) and found an ESKD prevalence of 1.5\% of almost 13,000 critically-ill surgical patients. The results showed that patients with ESKD had higher ICU mortality and hospital mortality than non-ESKD ( $23 \%$ vs $6 \%, \mathrm{p}<0.001$ and $31 \%$ vs $10 \%, \mathrm{p}<0.001$, respectively). These rates were quite similar to ours $(23 \%$ vs $5 \%, p=0.007$ and $34 \%$ vs $10 \%, p=0.002$, respectively. Moreover, the ICU length of stay was similar between the Apel M, et al study (2 days [IQR 1,7 days] vs 1 days [IQR 1,3 days], $\mathrm{p}<0.001$ ) (Apel et al. 2013) and ours (3 days [IQR 2,12 days] vs 2 days [IQR 1,6 days], $\mathrm{p}=0.007)$. Controversy regarding ESKD's association with poorer outcome in critically-ill patients was reported. Most of the studies confirmed ESKD as a predictor of poor prognosis for mortality 
251 among the critically ill (Hutchison et al. 2007; Manhes et al. 2005; Sood et al. 2011), although 252 some studies contradicted this (Strijack et al. 2009; Uchino et al. 2003). However, all of them 253 studied from mixed medical and surgical critically-ill patients, which might explain why outcomes 254 were inconsistent. outcomes among the critically-ill. In their multivariable analyses, ESKD was independently 257 associated with a greater risk of hospital mortality $(\operatorname{adjOR}=3.84,95 \% \mathrm{CI}, 2.68-5.50, \mathrm{p}<0.001$ when adjusted for age, gender, co-morbidities, SAPS II, type of surgery, and SOFA nonrenal score). In our study we identified quite similar features; ESKD was associated with both ICU and hospital mortality $(\operatorname{adjOR}=5.59 ; 95 \% \mathrm{CI}, 1.49-20.88, \mathrm{p}=0.01$ and $\operatorname{adjOR}=4.55 ; 95 \% \mathrm{CI}, 1.67-12.44$, $\mathrm{p}=0.003$, respectively; in ours, age and SOFA non-renal score were used for adjusting). data, which have rarely been reported. A trend for a higher grade of ASA physical status was found in our study. This may be explained by patients with ESKD are thought to be incapable and therefore expose themselves to greater risks from operative procedures than general surgical patients. The three most common surgical procedures in our study were vascular, cardiothoracic, and gastrointestinal surgery. Similarly, Apel M, et al reported cardiothoracic surgery as being the most common procedure (Apel et al. 2013). This corresponded to ESKD patients always having 269 cardiovascular problems due to comorbid diabetes mellitus and hypertension, which are most generally known as common causes of ESKD (Couser et al. 2011; Ong-Ajyooth et al. 2009). We found that sepsis and cardiovascular diseases were the first two common causes of our 272 ICU comorbidities, a finding noted in previous studies (Hutchison et al. 2007; Manhes et al. 2005; 273 Strijack et al. 2009). This means for patients already on long-term dialysis, more vital strategies 
274 should be focused upon, such as good pre-operative preparation, more invasive hemodynamic 275 monitoring, and optimal fluid therapy, among others.

276 In addition, due to a scarcity of evidence in critically-ill surgical patients with ESKD, the 277 mortality rates and its outcomes of ESKD and those of AKI were examined. The ICU mortality 278 from AKI in critically-ill surgical patients was greater in AKI than those non-AKI groups (26\% vs $2793 \%, \mathrm{p}<0.001)$ (Trongtrakul et al. 2019) and a greater hospital mortality (19\% vs 4\%; $\mathrm{p}=0.0001)$ 280 (Harris et al. 2015). Moreover, length of stay is longer when comparing the AKI to the non-AKI 281 groups. The studies from Trongtrakul K, et al (Trongtrakul et al. 2019) reported longer median 282 days in the ICU and the hospital (6 days [IQR 3,13 days] vs 1 days [ IQR 1,3 days], p $<0.001$ and 28318 days [IQR 10, 28 days] vs 14 days [IQR 9,24 days], $\mathrm{p}=0.003$, respectively). Longer median 284 days was also demonstrated in Harris DG, et al (Harris et al. 2015) (6 days [IQR 3-10 days] vs 3 285 days [IQR 2-5 days], $\mathrm{p}=0.001$ and 19 days [IQR 10-30 days] vs 9 days [IQR 5-16 days], $\mathrm{p}=0.0001$, respectively). Although there is a difference in the population studied, both the ESKD and the AKI 287 groups when undergoing a major operation and encountering a critical illness show a greater chance for mortality and longer length of stay in the ICU and in the hospital. Determining the different impact from ESKD and AKI in the critically-ill surgical patients is important for future 290 studies.

\section{Strengths}

Due to a lack of information regarding ESKD's effect on critically-ill surgical patients' outcomes, our study adds more information to this gap in knowledge. ESKD alone also has higher morbidity and mortality than the general population. In combination with major surgery, a known cause of overwhelming stress, this could lead to greater morbidity and mortality than among those 
298 serum creatinine level or urine output; in ESKD they are uncommonly low at baseline. Therefore,

299 SOFA non-renal score was also reported as one of the baseline characteristics. A priori matching

300 reduced differences between ESKD and non-ESKD participants. One might argue that propensity

301 score matching or a larger observational study should be done for exploring the hypothesis;

302 however, it was not possible in our setting, due to a lack of technology to support large data

303 collection. All patient data was manually extracted from the scanned medical records under our

304 best clinical practice and statistical methodology.

305 Limitations

306 There were some limitations in our study. Some information regarding ESKD was not collected,

307 for instance, the prevalence of ESKD in our cohort and pre-ICU admission data about ESKD

308 (e.g., dialysis vintage, dialysis adequacy, residual renal function). The other issue was a

309 limitation in multivariable logistic regression analysis. We would have liked to adjust

310 confounders that had an association with mortality. However, the low number of mortality

311 outcomes limits the number of variables included in the model. We are aware of

312 overparameterization when we included variables with more than one predictor per ten-event

313 outcomes. Therefore, the model was adjusted by a common demographic when patients were

314 admitted to the ICU, including age and SOFA non-renal score. Heterogeneity of type of

315 operation might be another interference on the outcome in our study. This should be investigated

316 in a larger scale study to identify pre-, peri-, and post-operative risk of mortality in surgical

317 critically-ill patients who underwent major operations. This could be useful in pre-operative

318 evaluation and management to improve this group of patient outcomes. Moreover, ESKD

319 patients, who exert a greater demand for ICU admission and a higher chance of mortality, should 
320 be fully evaluated for the factors that have an impact on surgical ICU performance, for better and

321 more appropriate resource allocation and management.

322 Conclusion

323

324

325

326

327

328

329

330

331

332

333

334

335

336

337

338

339

340

341

342

Critically-ill surgical patients with ESKD were strongly associated with higher mortality compared to non-ESKD patients. Besides appropriate surgical wound and drainage care, invasive monitoring to understand the complexity of the patient volume status and scheduling adequate dialysis to maintenance patient metabolic and electrolyte homeostasis are encouraged. Moreover, to achieve the most favorable outcome for critically-ill surgical patients with ESKD who undergo a major operation, acute care must be endorsed throughout the pre-, peri-, and postoperative period.

\section{Acknowledgements}

We would like to acknowledge the Faculty of Medicine Vajira Hospital,

Navamindradhiraj University for supporting our work and providing information. We also would like to kindly thank Mr. Jason D. Cullen and Mr. Eric B. Tedstrom, MSW who helped to review and edit our manuscript.

\section{References}

Apel M, Maia VP, Zeidan M, Schinkoethe C, Wolf G, Reinhart K, and Sakr Y. 2013. End-stage renal disease and outcome in a surgical intensive care unit. Crit Care 17:R298. $10.1186 /$ cc13167 
343 Arulkumaran N, Annear NM, and Singer M. 2013. Patients with end-stage renal disease

344 admitted to the intensive care unit: systematic review. Br J Anaesth 110:13-20.

$345 \quad 10.1093 /$ bja/aes401

346 Deng Y, Yuan J, Chi R, Ye H, Zhou D, Wang S, Mai C, Nie Z, Wang L, Zhai Y, Gao L, Zhang D, Hu L, Deng Y, and Chen C. 2017. The Incidence, Risk Factors and Outcomes of

Elia C, Schoenfeld C, Bayer O, Ewald C, Reinhart K, and Sakr Y. 2013. The impact of age on Postoperative Acute Kidney Injury in Neurosurgical Critically III Patients. Sci Rep 7:4245. outcome after major surgical procedures. J Crit Care 28:413-420. 10.1016/j.jcrc.2012.12.010

Fidalgo P, and Bagshaw SM. 2014. Chronic Kidney Disease in the Intensive Care Unit. Management of Chronic Kidney Disease: A Clinician's Guide:417-438. 10.1007/978-3$642-54637-2 \_32$

Forouzanfar MH, Liu P, Roth GA, Ng M, Biryukov S, Marczak L, Alexander L, Estep K, Hassen 357 Abate K, Akinyemiju TF, Ali R, Alvis-Guzman N, Azzopardi P, Banerjee A, Barnighausen T, Basu A, Bekele T, Bennett DA, Biadgilign S, Catala-Lopez F, Feigin VL, Fernandes JC, Fischer F, Gebru AA, Gona P, Gupta R, Hankey GJ, Jonas JB, Judd SE, Khang YH, Khosravi A, Kim YJ, Kimokoti RW, Kokubo Y, Kolte D, Lopez A, Lotufo PA, Malekzadeh R, Melaku YA, Mensah GA, Misganaw A, Mokdad AH, Moran AE, Nawaz H, Neal B, Ngalesoni FN, Ohkubo T, Pourmalek F, Rafay A, Rai RK, Rojas-Rueda D, Sampson UK, Santos IS, Sawhney M, Schutte AE, Sepanlou SG, Shifa GT, Shiue I, Tedla BA, Thrift AG, Tonelli M, Truelsen T, Tsilimparis N, Ukwaja KN, Uthman OA, Vasankari T, Venketasubramanian N, Vlassov VV, Vos T, Westerman R, Yan LL, Yano Y, Yonemoto N, Zaki ME, and Murray CJ. 2017. Global Burden of Hypertension and Systolic Blood Pressure of at Least 110 to $115 \mathrm{~mm} \mathrm{Hg}, 1990-2015$. JAMA 317:165-182. 10.1001/jama.2016.19043 
369 Geiss LS, Wang J, Cheng YJ, Thompson TJ, Barker L, Li Y, Albright AL, and Gregg EW. 2014. $370 \quad$ Prevalence and incidence trends for diagnosed diabetes among adults aged 20 to 79 years, United States, 1980-2012. JAMA 312:1218-1226. 10.1001/jama.2014.11494

372 Goulding MR, Rogers ME, and Smith SM. 2003. From the Centers for Disease Control and 373 Prevention. Public health and aging: trends in aging--United States and worldwide. $374 \quad$ JAMA 289:1371-1373.

375 Hales CM, Fryar CD, Carroll MD, Freedman DS, and Ogden CL. 2018. Trends in Obesity and 376 Severe Obesity Prevalence in US Youth and Adults by Sex and Age, 2007-2008 to 377 2015-2016. JAMA 319:1723-1725. 10.1001/jama.2018.3060

378 Harris DG, McCrone MP, Koo G, Weltz AS, Chiu WC, Scalea TM, Diaz JJ, and Lissauer ME. 2015. Epidemiology and outcomes of acute kidney injury in critically ill surgical patients. J Crit Care 30:102-106. 10.1016/j.jcrc.2014.07.028

Hasan I, Bahalkeh E, and Yih Y. 2020. Evaluating intensive care unit admission and discharge 382 policies using a discrete event simulation model. SIMULATION 96:501-518. $10.1177 / 0037549720914749$

384 385 386 387 388 389 390 391 392 393

McCullough KP, Morgenstern H, Saran R, Herman WH, and Robinson BM. 2019. Projecting ESRD Incidence and Prevalence in the United States through 2030. J Am Soc Nephrol 30:127-135. 10.1681/ASN.2018050531

Pornwaragron C, Wilasrusmee C, Morakul C, Poprom N, and Horsirimanont S. 2019. Application of APACHE-II and SOFA score as a Predictive Outcome in Ramathibodi Surgical Intensive Care Unit. J Med Assoc Thai 102:80.

Praditpornsilpa K, Lekhyananda S, Premasathian N, Kingwatanakul P, Lumpaopong A, Gojaseni P, Sakulsaengprapha A, Prasithsirikul W, Phakdeekitcharoen B, Lelamali K, Teepprasan T, Aumanaphong C, Leerawat B, Pongpiyadej J, Srangsomvong S, Kanjanabuch T, Eiam-Ong S, Vareesaengthip K, and Lumlertkul D. 2011. Prevalence 
394

395

396

397

398

399

400

401

402

403

404

405

406

407

408

409

410

411

412

413

414

415

416

trend of renal replacement therapy in Thailand: impact of health economics policy. $J$ Med Assoc Thai 94 Suppl 4:S1-6.

Romo H, Amaral AC, and Vincent JL. 2004. Effect of patient sex on intensive care unit survival. Arch Intern Med 164:61-65. 10.1001/archinte.164.1.61

Steyerberg EW, Schemper M, and Harrell FE. 2011. Logistic regression modeling and the number of events per variable: selection bias dominates. J Clin Epidemiol 64:1464-1465; author reply 1463-1464. 10.1016/j.jclinepi.2011.06.016

Trongtrakul K, Sawawiboon C, Wang AY, Chitsomkasem A, Limphunudom P, Kurathong S, Prommool S, Trakarnvanich T, and Srisawat N. 2019. Acute kidney injury in critically ill surgical patients: Epidemiology, risk factors and outcomes. Nephrology (Carlton) 24:3946. $10.1111 /$ nep. 13192

van Sluisveld N, Oerlemans A, Westert G, van der Hoeven JG, Wollersheim H, and Zegers M. 2017. Barriers and facilitators to improve safety and efficiency of the ICU discharge process: a mixed methods study. BMC health services research 17:251-251. $10.1186 / \mathrm{s} 12913-017-2139-x$

Wald R, McArthur E, Adhikari NK, Bagshaw SM, Burns KE, Garg AX, Harel Z, Kitchlu A, Mazer CD, Nash DM, Scales DC, Silver SA, Ray JG, and Friedrich JO. 2015. Changing incidence and outcomes following dialysis-requiring acute kidney injury among critically ill adults: a population-based cohort study. Am J Kidney Dis 65:870-877. 10.1053/j.ajkd.2014.10.017

Walter S, and Tiemeier H. 2009. Variable selection: current practice in epidemiological studies. Eur J Epidemiol 24:733-736. 10.1007/s10654-009-9411-2 
Figure 1

Flow of the study 


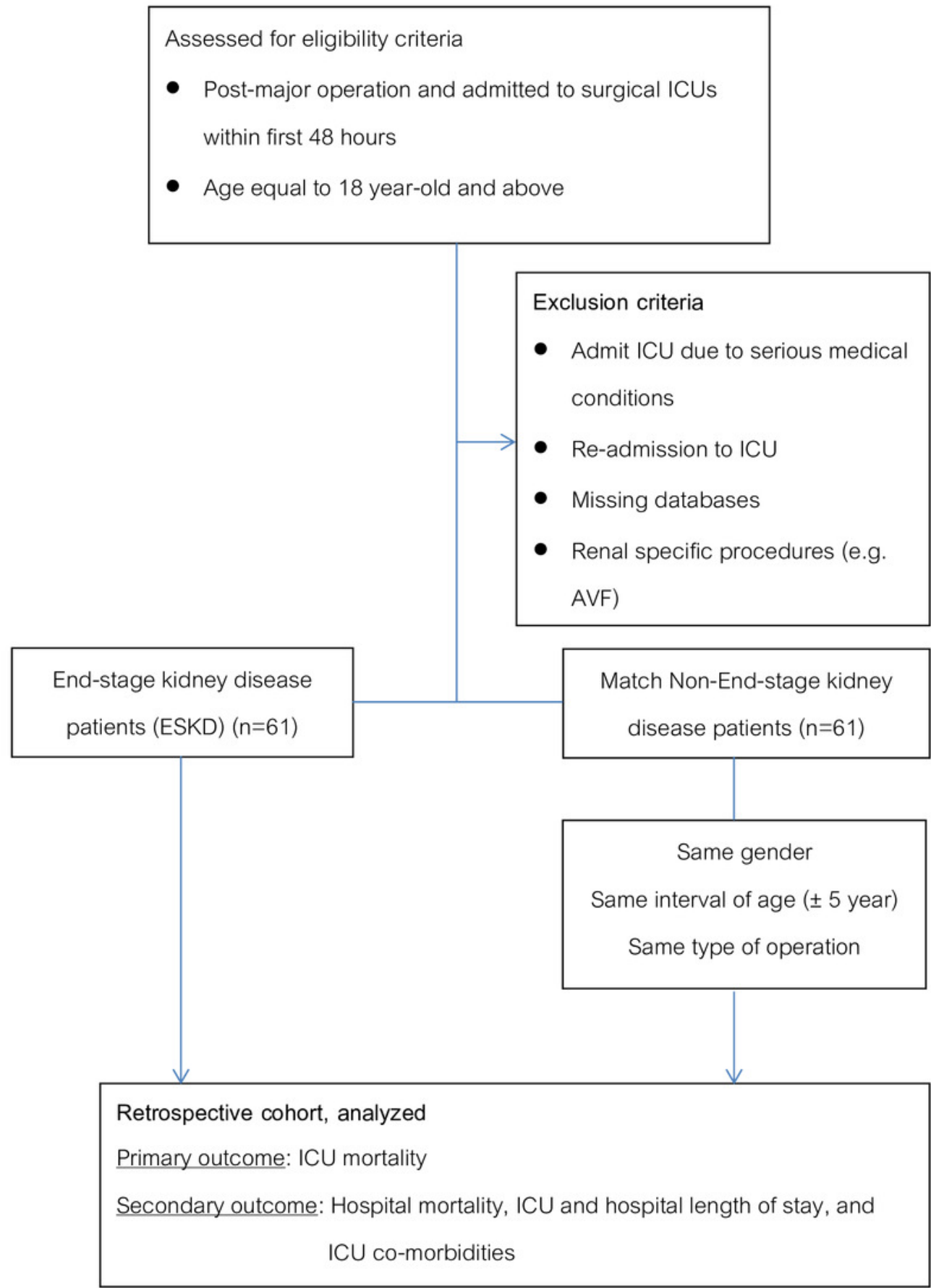


Figure 2

Mortality rates between critically-ill surgical patients with ESKD and non-ESKD populations.

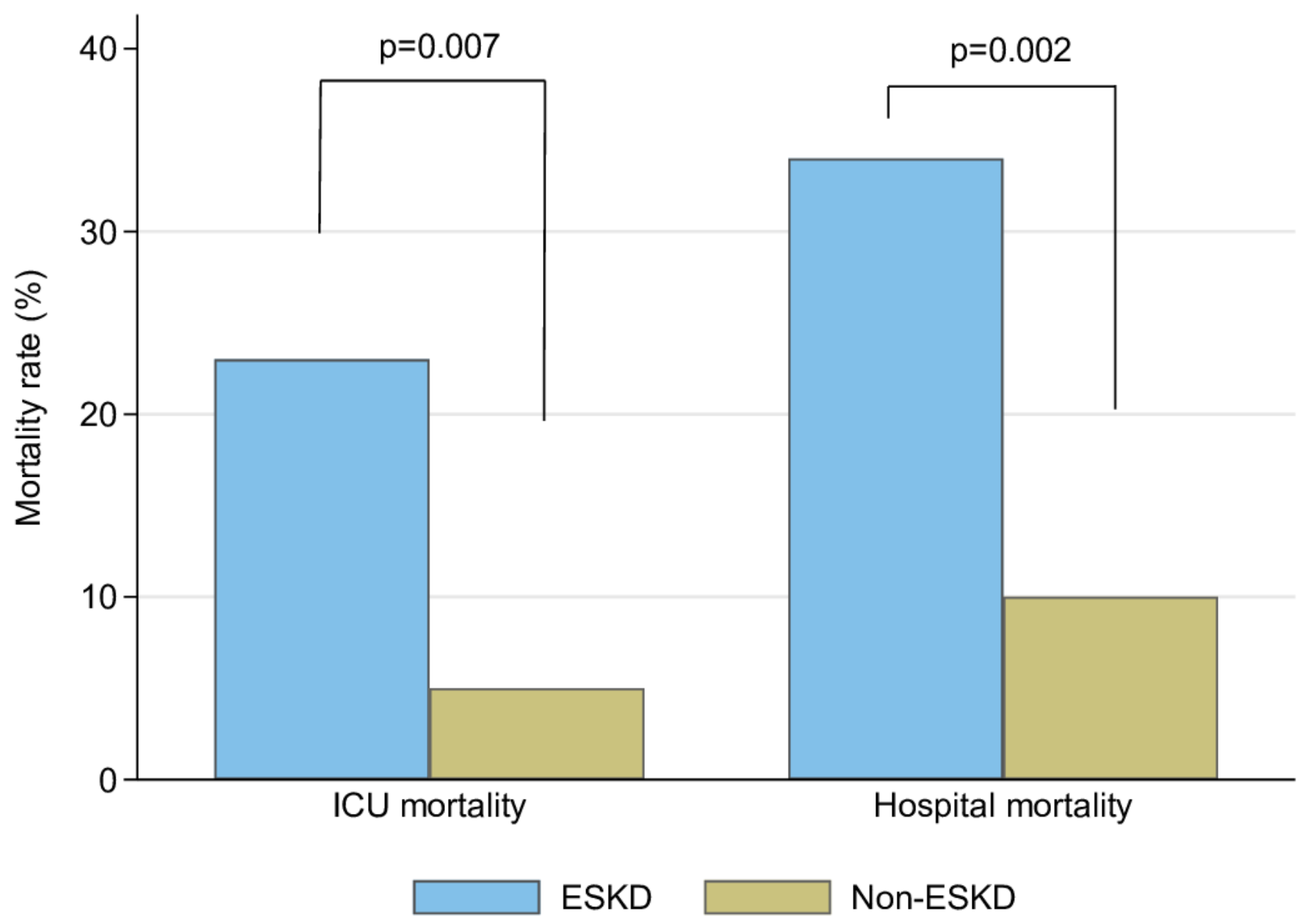




\section{Table $\mathbf{1}$ (on next page)}

Baseline characteristics of the study group on admission to the intensive care unit according to the presence of end-stage kidney disease (ESKD) and non-ESKD.

*Reported as median and Interquartile range 1 and 3; ASA, the American Society of Anesthesiologist; ICU, intensive care unit; SOFA score, the Sequential Organ Failure Assessment score. 


\begin{tabular}{|c|c|c|c|}
\hline Characteristics & $\begin{array}{l}\text { ESKD } \\
(\mathrm{n}=61)\end{array}$ & $\begin{array}{c}\text { Non-ESKD } \\
(\mathrm{n}=61)\end{array}$ & P-value \\
\hline Age - years & $67.5 \pm 10.1$ & $66.8 \pm 10.6$ & 0.71 \\
\hline Male - n (\%) & $35(57 \%)$ & $36(59 \%)$ & $>0.99$ \\
\hline Body weight - kg & $58.5 \pm 11.4$ & $60.7 \pm 11.7$ & 0.30 \\
\hline Height - cm & $161 \pm 8$ & $160 \pm 8$ & 0.26 \\
\hline Body mass index $-\mathrm{kg} / \mathrm{sq}-\mathrm{m}$ & $23.7 \pm 3.8$ & $22.6 \pm 4.8$ & 0.16 \\
\hline \multicolumn{4}{|l|}{ Pre-existing conditions, $\mathrm{n}(\%)$} \\
\hline Diabetes mellitus & $41(67 \%)$ & $28(46 \%)$ & 0.03 \\
\hline Hypertension & $54(89 \%)$ & $44(72 \%)$ & 0.04 \\
\hline Hyperlipidemia & $20(33 \%)$ & $22(36 \%)$ & 0.85 \\
\hline Cardiovascular disease & $23(38 \%)$ & $20(33 \%)$ & 0.71 \\
\hline Cerebrovascular accident & $10(16 \%)$ & $6(10 \%)$ & 0.42 \\
\hline Chronic kidney disease & - & $7(12 \%)$ & - \\
\hline End stage kidney disease & $61(100 \%)$ & - & - \\
\hline ASA classification, n (\%) & & & $<0.001$ \\
\hline 2 & $0(0 \%)$ & $12(20 \%)$ & \\
\hline 3 & $52(85 \%)$ & $45(74 \%)$ & \\
\hline 4 & $9(15 \%)$ & $4(6 \%)$ & \\
\hline Anesthesia technique, $\mathrm{n}(\%)$ & & & 0.49 \\
\hline General anesthesia & $55(90 \%)$ & $58(95 \%)$ & \\
\hline Spinal block & $6(10 \%)$ & $3(5 \%)$ & \\
\hline Emergency surgery, n (\%) & $26(43 \%)$ & $23(38 \%)$ & 0.71 \\
\hline \multicolumn{4}{|l|}{ Types of surgery, n (\%) } \\
\hline Gastrointestinal surgery & $8(13 \%)$ & $17(28 \%)$ & 0.07 \\
\hline Vascular surgery & $21(34 \%)$ & $11(18 \%)$ & 0.06 \\
\hline Urology & $7(12 \%)$ & $7(11 \%)$ & $>0.99$ \\
\hline Hepato-pancreato-biliary & $2(3 \%)$ & $2(3 \%)$ & $>0.99$ \\
\hline Neurosurgery & $1(2 \%)$ & $1(2 \%)$ & $>0.99$ \\
\hline Cardiovascular thoracic & $13(21 \%)$ & $14(23 \%)$ & $>0.99$ \\
\hline Others & $9(15 \%)$ & $9(15 \%)$ & $>0.99$ \\
\hline Operative time (hours)* & $4(2,6)$ & $3.5(2,5)$ & 0.34 \\
\hline \multicolumn{4}{|c|}{ Illness severity at ICU admission } \\
\hline SOFA & $8.9 \pm 2.6$ & $5.6 \pm 2.5$ & $<0.001$ \\
\hline SOFA non renal & $5.7 \pm 2.2$ & $5.2 \pm 2.3$ & 0.16 \\
\hline
\end{tabular}

1

2

3

4

5

6

7

8 


\section{Table 2 (on next page)}

Morbidity and mortality of the study group according to the presence of end-stage kidney disease.

*Reported as median and interquartile range 1 and 3; ICU, intensive care unit. 


\begin{tabular}{llll}
\hline Outcomes & $\begin{array}{l}\text { ESKD } \\
(\mathrm{n}=61)\end{array}$ & $\begin{array}{l}\text { Non-ESKD } \\
(\mathrm{n}=61)\end{array}$ & P-value \\
\hline Primary outcomes & $14(23 \%)$ & $3(5 \%)$ & 0.007 \\
ICU mortality, n (\%) & $21(34 \%)$ & $6(10 \%)$ & 0.002 \\
Secondary outcomes & $3(2,12)$ & $2(1,6)$ & 0.007 \\
Hospital mortality, n (\%) & $29(16,51)$ & $20(14,34)$ & 0.03 \\
ICU length of stay - days* & & & \\
Hospital length of stay - days* & $24(39 \%)$ & $23(38 \%)$ & $>0.99$ \\
ICU co-morbidities, n (\%) & $11(18 \%)$ & $9(15 \%)$ & 0.81 \\
Severe sepsis/septic shock & $7(11 \%)$ & $14(23 \%)$ & 0.22 \\
$\quad$ Pneumonia & $19(31 \%)$ & $15(25 \%)$ & 0.55 \\
$\quad$ Surgical site infection & $11(18 \%)$ & $4(7 \%)$ & 0.10 \\
Cardiovascular diseases & $7(11 \%)$ & $7(11 \%)$ & $>0.99$ \\
$\quad$ Acute coronary syndrome & $4(7 \%)$ & $8(13 \%)$ & 0.36 \\
Congestive heart failure & $2(3 \%)$ & $3(5 \%)$ & $>0.99$ \\
Cardiac arrhythmia & $3(5 \%)$ & $1(2 \%)$ & 0.62 \\
Gastrointestinal bleeding & $1(2 \%)$ & $0(0 \%)$ & $>0.99$ \\
Cerebrovascular accident & $9(15 \%)$ & $12(20 \%)$ & 0.63 \\
Deep venous thrombosis & &
\end{tabular}

1 


\section{Table 3(on next page)}

Multivariable regression analyses the association of ESKD affected on ICU mortality and hospital mortality.

*Adjusted by age and SOFA non-renal score. 


\begin{tabular}{llllrll}
\hline & OR & $95 \%$ CI & P value & AdjOR* & $95 \%$ CI & P value \\
\hline ICU mortality & 5.76 & 1.56 to 21.24 & 0.009 & 5.59 & $1.49-20.88$ & 0.01 \\
Hospital mortality & 4.81 & 1.78 to 13.01 & 0.002 & 4.55 & $1.67-12.44$ & 0.003 \\
\hline
\end{tabular}

